\title{
Comparison of Fascia lliaca Block with Intravenous Paracetamol, as Postoperative Analgesic Method for Femur Surgery
}

\author{
Praveenkumar Shekhrajka', Dhavalkumar C Patel ${ }^{2}$ \\ ${ }^{1}$ Consultant anesthesiologist, IBS Jaipur, Rajasthan, India, ${ }^{2}$ Assistant Professor, GMC Surat, Gujarat, India.
}

\section{Abstract}

Background: Postoperative pain is extremely unpleasant which causes severe discomfort to the patient as well as it increases the anxiety, hospital stay so overall total burden. Objectives: Comparison of efficacy between fascia iliaca block with intravenous paracetamol, as postoperative analgesic method after femur surgery. Simultaneously requirement of other analgesics in the first 24 hours. Subjects and Methods: Prospective longitudinal study conducted in patients belonging to age group of 18 to 65 years posted for femur surgeries. All patients were received Intrathecal injection of $3.5 \mathrm{ml}$ of $0.5 \%$ hyperbaric bupivacaine and divided into two groups. After completion of surgery Group I received the Fascia Iliaca block with thirty $\mathrm{ml}$ of $0.25 \%$ of injection bupivacaine and Group II received injection Paracetamol one gram intravenously. Duration of analgesia, time from block / iv PCM to 1st rescue analgesia, number of patients as well as total doses of required rescue analgesics in 1st 24 hours were observed postoperatively. Results: From One hour onwards at all the time interval, mean pain scores was less in FIB group as compared to PCM group(p<0.001). In FIB group $15(42 \%)$ patients had more than 24 hours and $11(32 \%)$ patients had 12-24 hours of analgesia, where as in PCM Group not a single patient had more than 12 hours of analgesia. In FIB group only 20 (57\%) patients required rescue analgesic as compared to $35(100 \%)$ patients in PCM group. Conclusion: In FIB insertion point is away from femoral vessels and can be provided without nerve stimulator. Post-operative it is extremely crucial to provide analgesia specially initial 24 hours. FIB can provide prolong duration of analgesia, so helps in early mobilization and early rehabilitation as well as it also reduces opioid requirements. Thus, Fascia iliaca block is an effective, easy and affordable method for postoperative analgesia and can be used safely as a part of multimodal approach to pain relief after femur surgery.

Keywords: Postoperative analgesia, femur surgery, Fascia Iliaca block (FIB), PCM (Paracetamol), Simplified Verbal Scale scores (SVS score).

Corresponding Author: Dr. Dhavalkumar C Patel, Department of Anaesthesia GMC, Surat, Gujarat, India.

Email: dcp991717@gmail.com

Received: January 2020

Accepted: January 2020

\section{Introduction}

Post-operative analgesic regimen can be labelled as ideal when it can provide effective pain relief, affordable to all, minimally invasive and without side-effects.

When we ignore Postoperative pain, it leads to various physiological and psychological dysfunctions in the body which may ultimately increase the incidence of morbidity and mortality. Hence to treat postoperative pain is not only humanitarian but also necessary to reduce postoperative sickness. ${ }^{[1]}$ Postoperative pain is extremely unpleasant which causes severe discomfort to the patient as well as it increases the anxiety, hospital stay so overall total burden to all. ${ }^{[2]}$

For effective pain relief we have to provide satisfactory postoperative pain relief without sedation. Parenteral opioids are widely used for post-operative pain relief by consultants. Parenteral route for opioids is associated with many complications like vomiting, constipation, respiratory depression and for long term use addiction etc. Techniques like patient-controlled analgesia and epidural analgesia require specific devices, knowledge to handle it and dedicated staff which is overall costly. ${ }^{[3]}$

Regional anaesthesia has many advantages over general anaesthesia with low incidence of sedation, nausea, vomiting and post-operative respiratory events. As the effect of peripheral neural block gradually dissipates it can provide smooth transition to pain control, ultimately leads to early discharge from recovery room. Neural blockade provides pre-emptive analgesia by preventing the nociceptive input to the spinal cord thereby reduces the postoperative pain, analgesic usage and hence early discharge. Regional blocks have wide application in providing surgical anaesthesia and analgesia as well as in treating chronic pain syndrome involving the extremities. ${ }^{[4,5]}$

A number of additives like morphine, pethidine, butorphanol, tramadol, clonidine, dexmedetomidine, midazolam can be used in peripheral nerve block with local anesthetic agents to prolong the duration of analgesia which can reduce large dose of local anesthetics and ultimately the adverse effects. ${ }^{[6]}$ But here we want to know the effect of block versus conventional pain relief strategies, so we have not added any 
adjuvants to prevent bias.

Fracture lower limb bone specially femur is a common injury at the age around 50-70 years which causes extremely distress and pain to the patient. Fracture femur may involve femoral neck or femoral shaft or distal femur. ${ }^{[7]}$ Analgesia is provided by conventional modes of pain relief like nonsteroidal anti-inflammatory drugs, opioids, etc. It can be provided by peripheral nerve blocks like the femoral nerve block, 3 in 1 block, the Fascia Iliaca block (FIB) and lumbar plexus block. There are few data available and not commonly practiced at our institute regularly so to know the benefit of one form of analgesia over another, the need of this study arises. So, we are planning to do this study to evaluate the "Comparison of fascia iliaca block with intravenous paracetamol, as postoperative analgesic method for femur surgery.

\section{Aim Of Our Study:}

Comparison of fascia iliaca block with intravenous paracetamol, as postoperative analgesic method for femur surgery. Simultaneously compared the efficacy of Fascia Iliaca Block using local anesthetic bupivacaine versus intravenous paracetamol and the requirement of other analgesics in the first 24 hours post operatively in both groups.

\section{Subjects and Methods}

After getting approval from institutional ethical committee in 2016 seventy patients scheduled for elective or emergency femur surgeries were included in the study with their written consent. This is prospective single-blinded longitudinal study conducted in patients belonging to ASA grade I to III, posted for elective or emergency surgeries of either sex in the age group of 18 to 65 years. Patients who were not following inclusion criteria, apart from femur other bone fracture/other major injuries, with known hypersensitivity or contraindications to local anesthetics and with supplemented epidural or general anesthesia were excluded from study.

Total of 70 patients who were scheduled for elective or emergency femur surgery were randomly divided by computer generated number into two groups by consultant anesthesiologist in FIB (Fascia Iliaca block) Group \& PCM (Paracetamol) Group with comprising of 35 patients in each group. All patients were received Intrathecal injection of 3.5 $\mathrm{ml}$ of $0.5 \%$ hyperbaric bupivacaine in sitting or lateral position with $23 \mathrm{G} / 25 \mathrm{G}$ spinal needle under strict aseptic precaution. After completion of surgery when the sensory level of Sub-arachnoid block regressed to L1 the study was started in post-operative recovery room.

Hemodynamic baseline parameters like heart rate, noninvasive blood pressure and saturation of oxygen ( $\mathrm{SpO} 2)$, respiratory rate was recorded in all individuals. Group I (FIB) received the Fascia Iliaca block with thirty $\mathrm{ml}$ of $0.25 \%$ of Injection bupivacaine, after completion of surgery. Group II (PCM) received Injection Paracetamol, one gram, intravenously after completion of surgery. Following parameters were observed in our study: Duration of analgesia -time from block or iv PCM to 1st rescue analgesia, Number of patients required rescue analgesic in 24 hours postoperatively, total dose of rescue analgesic needed in 24 hours and for any side effect.

The analgesia provided by either of the modes was subjectively assessed by using Simplified Verbal Scale scores (SVS score) mentioned as 0- No pain, 1-Mild pain, 2Moderate pain, 3-Severe pain, 4-Extreme pain. Post operatively patients were asked to grade their pain with SVS and SVS score was noted at 10 minutes, 20 minutes, 30 minutes, $1^{\text {st }}$ hour, $2^{\text {nd }}$ hour, $4^{\text {th }}$ hour, $6^{\text {th }}$ hour, $12^{\text {th }}$ hour and then at $24^{\text {th }}$ hour.

Analgesia was given in form of Injection Tramadol $2 \mathrm{mg} / \mathrm{kg}$. First dose was given whenever patient complain of SVS $>2$. After that analgesia in form of Tramadol was repeated every 8 hours. After routine analgesic dose, if still pain persists, Injection Diclofenac, 75mg intra-muscularly, (on request by the patient) was administered and total dose of analgesic in 24 hours was noted.

\section{Statistical Analysis:}

All data were analyzed using EPI 2000 software. All the data were presented as mean and standard deviation (SD), except where specified. For continuous variable ' $t$ ' test was used and for non-continuous variable chi test was used, $\mathrm{p}>0.05$ was considered as non-significant, $\mathrm{p}<0.05$ was considered statistically significant and $\mathrm{p}<0.001$ considered as highly significant. ${ }^{[8]}$

\section{Results \& Discussion}

Fracture femur is most common injury of lower limb at elderly. In present study Mean age of patients in FIB group was 54.4 \pm 11.6 years while in PCM group it was 51.69 \pm 13.44 years $(\mathrm{p}>0.05)$. Mean weight of patients in FIB group was $54.43 \pm 9.21 \mathrm{Kg}$ while in PCM group it was $54 \pm 8.03 \mathrm{Kg}$ (p> $0.05)$. Thus, both the groups were comparable with respect to age and weight.

All patients were received Intrathecal injection of $3.5 \mathrm{ml}$ of $0.5 \%$ hyperbaric bupivacaine in sitting or lateral position with $23 \mathrm{G} / 25 \mathrm{G}$ spinal needle under strict aseptic precaution. After completion of surgery when the sensory level of Subarachnoid block regressed to L1 the study was started. Haddad F S et al (2015), ${ }^{[9]}$ conducted a study to evaluate the analgesic efficacy of femoral nerve block and systemic analgesic alone on extra capsular femur neck fracture. Fentahun T K et al (2015), ${ }^{[10]}$ conducted a study to evaluate the efficacy of Fascia iliaca compartment block as part of multimodal analgesia after surgery for femoral bone fracture using $0.25 \%$ bupivacaine.

Hemodynamic baseline parameters like heart rate, noninvasive blood pressure were recorded in all individuals. At baseline there was no statistical difference in pulse rate in FIB Group and PCM Group. Although, after subsequent recording of pulse rate at 1 Hour, 2 Hour and at 4 Hour there was statistically significant different $(\mathrm{p}<0.05)$. Because FIB has long term analgesic effect while, PCM has short time effect. So, when effect of PCM subsides there was difference in pulse rate in this study.

There was no statistical difference in Systolic Blood Pressure (SBP) and Diastolic Blood Pressure (DBP) in FIB Group and PCM Group at baseline. Although, at 1 Hour and 2 Hour of Systolic Blood Pressure recording there was statistically significant difference $(\mathrm{p}<0.05)$. And for Diastolic Blood 
Pressure at 1 Hour there was statistically significant difference noted $(\mathrm{p}<0.05)$. But both SBP and DBP changes were clinically within physiological limit.

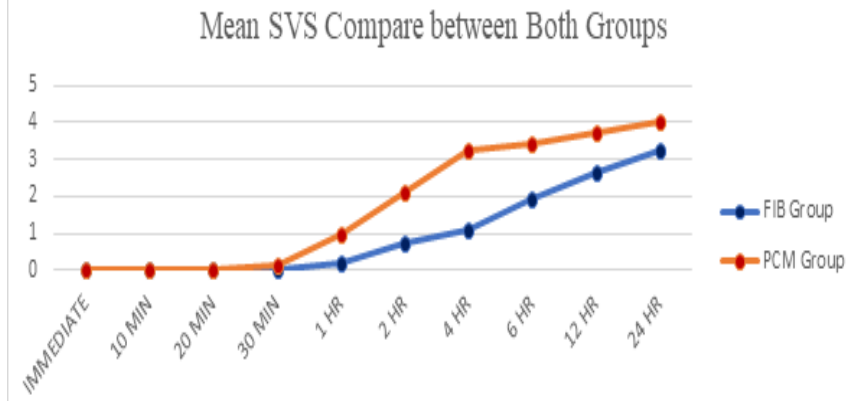

Figure 1: Mean Svs Score At Different Time Intervals In Both Groups

[Figure 1] shows mean SVS score at various time intervals in both the groups. Up to the 30 minutes, SVS scores was zero in both the groups. From One hour onwards at all the time interval, mean SVS scores was less in FIB group as compared to PCM group. At 1 st hour ( 0.18 vs 0.97$)$, at 2 nd hour $(0.71$ vs 2.11$)$, at $4^{\text {th }}$ hour (1.11 vs 3.26$)$, at 6 th hour (1.96 vs 3.43$)$, at 12 th hour (2.64 vs 3.71$)$, at $24^{\text {th }}$ hour $(3.25$ vs 4$)$ and these differences were statistically significant $(\mathrm{p}<0.001)$.

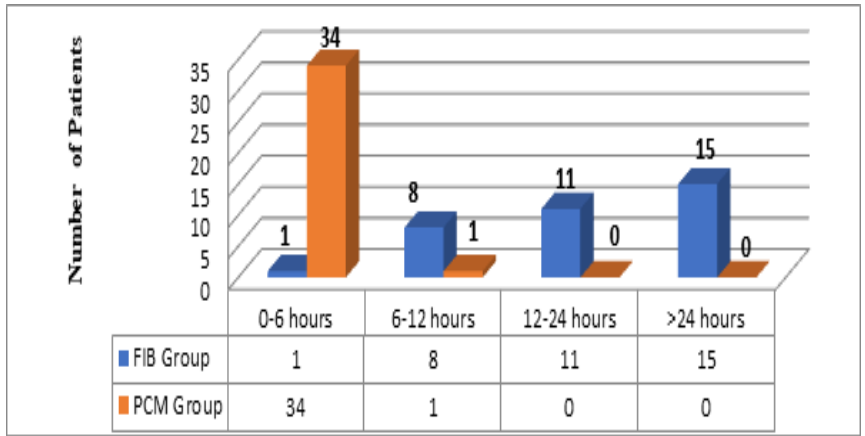

Figure 2: Duration Of Analgesia Among Patients In Both The Groups

For assessment of pain Simplified Verbal Scale Score (SVS) (0 - 4, 0-no pain to 4-extreme pain) was used. When patient achieved SVS $>2$, Injection Tramadol $2 \mathrm{mg} / \mathrm{kg}$ was given intravenously as rescue analgesia 8 hourly and even if pain persisted, injection Diclofenac $75 \mathrm{mg}$ intramuscularly was administered.

In FIB group out of 35 patients, 15 (42\%) patients had more than 24 hours of analgesia. 11(32\%) patient had 12-24 hours of analgesia, $8(23 \%)$ patients had 6-12 hours of analgesia and $1(3 \%)$ had analgesia up to 6 hours. [Figure 2]

Where as in PCM Group out of 35 patients, not a single patient had either more than 24 hours of analgesia or 12-24 hours of analgesia. 1 (3\%) patient had 6-12 hours of analgesia and 34(97\%) had analgesia up to 6 hours. Hanna L et al (2014), ${ }^{[11]}$ conducted a study on the role of fascia iliaca blocks in hip fractures. There was significant reduction in pain score which continued up to 8 hours $(\mathrm{p} \leq 0.05)$ in FIB group. Fentahun T K et.al (2015), ${ }^{[10]}$ conducted a study to evaluate the efficacy of Fascia iliaca compartment block as part of multimodal analgesia after surgery for femoral bone fracture using $0.25 \%$ bupivacaine. They have noted FICB group had a longer time for the first analgesic request than in control group. [(mean \pm SD) FICB $(417 \pm 112.10)$ vs $(139.25$ $\pm 37.67)$ minutes in another group.]

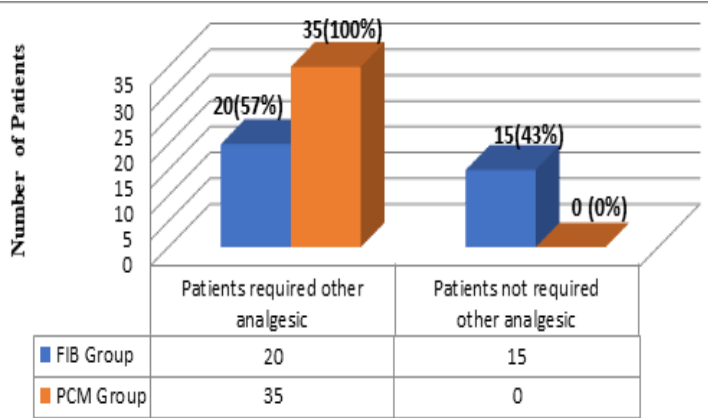

Figure 3: Total Number Of Patients Require Rescuse Analgesic In Both The Groups

In FIB group only $20(57 \%)$ patients required rescue analgesic as compared to $35(100 \%)$ patients in PCM group. Large proportion of patients in FIB group did not require any analgesic as compared to PCM group. In FIB group number of patients who required rescue analgesic were statistically significant less as compared to PCM group. ( $\mathrm{p}<0.0001)$. [Figure 3]. Hanna L et al (2014), ${ }^{[11]}$ conducted a study on the role of fascia iliaca blocks in hip fractures. FIB patients required low doses of systemic analgesia. Nie $\mathrm{H}$ et al (2015), ${ }^{[12]}$ conducted a study on efficacy of continuous fascia iliaca compartment block (FIB) with Patient-Controlled Intravenous Analgesia (PCIA) as postoperative analgesia after hip fracture surgery. In FIB group 39.2\% patients received additional analgesia compare to $41.5 \%$ patients in PCIA group. Williams $\mathrm{H}$ et al (2016),${ }^{[13]}$ conducted a study on standard pre-operative analgesia with or without fascia iliaca compartment block (FICB) for femoral neck fracture. $38 \%$ patients with standard analgesia and with FICB required no additional opioids analgesia.

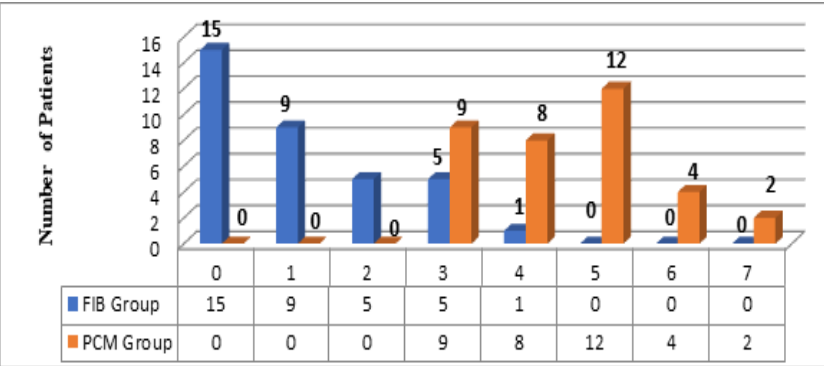

Figure 4: Doses (Numbers) Of Rescue Analgesic Required Among Both Groups

In our study we observed that in FIB group among the patients, who required other analgesic (total 20 patients), 9 patients required only 1 dose, 5 patients required 2 doses, 5 patients required 3 doses and 1 patient required 4 doses. In PCM group among the patients, all patients required other analgesic (total 35 patients), 9 patients required 3 doses, 8 patients required 4 doses, 12 patients required 5 doses, 4 patients required 6 doses and 2 patients required 7 doses. [Figure 4]. Williams $\mathrm{H}$ et al (2016), ${ }^{[13]}$ conducted, additional of FICB reduced the mean dose of opioid from 6.2 to 2.0 and the number of opioid overdoses from $7.2 \%$ to $0 \%$, compared 
with standard analgesia alone.

In FIB group 30 tramadol $(2 \mathrm{mg} / \mathrm{kg})$ and 8 diclofenac $(75 \mathrm{mg})$ doses required as compared to 103 tramadol and 54 diclofenac doses in PCM group. [Figure 5]

None of the patients in any group had any complication. Nicolai B et al (2007), ${ }^{[14]}$ and Williams $\mathrm{H}$ et al (2016), ${ }^{[13]}$ also did not observed any side effect of the FICB technique.

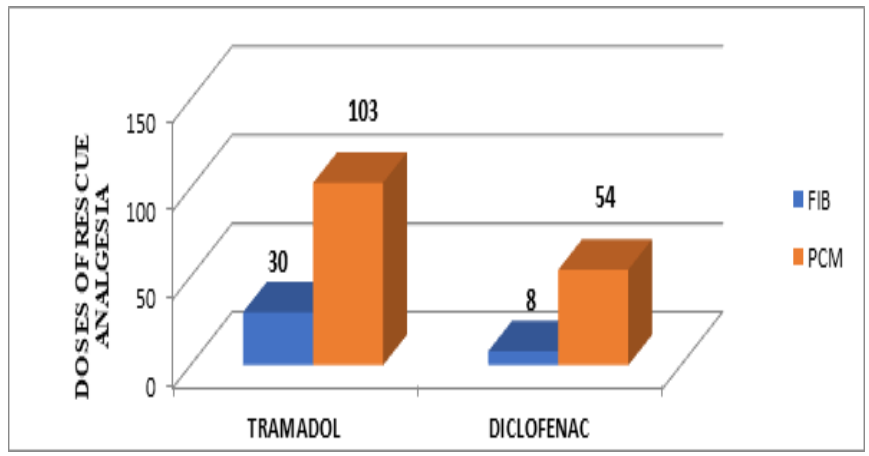

Figure 5: Different Doses (Numbers) Of Rescue Analgesic Required Among Both Groups

\section{Conclusion}

It was concluded from above observation that Fascia liaca compartment block (FICB) is neural block of anterior thigh of lumbar plexus. As it is injected into fascia iliaca compartment, it spread to femoral and lateral cutaneous nerve of thigh and then proximally and medially to enter space of psoas and iliacus muscle reaching obturator nerve in one third of cases. FICB is superior to femoral nerve block because nerve stimulator is not required and it is easy, safe to perform and insertion point is away from femoral nerve and vessel. It reduces opioid requirement by $66 \%$ and less side effects like nausea, vomiting and respiratory depression. Prolonged duration of analgesia, early mobilization and early rehabilitation. SVS score less than 3 up to 24 hours postoperatively. Minimum requirement of rescue analgesic during initial 24 hours postoperatively.

Thus, we can conclude that Bupivacaine $(0.25 \%)$, when given as Fascia iliaca block is an effective, easy and affordable method for postoperative analgesia and can be used safely as a part of multimodal approach to pain relief after femur surgery.

\section{Strength of The Study:}

Fascia iliaca block does not require much skill or specialized equipment so it easy to administer. Less number of nursing staff are required postoperatively to manage the patient as duration of action of Fascia iliaca block of bupivacaine is prolonged. Fascia iliaca block reduce overall pain and hospital stay. No, side effects are observed, so no close monitoring is required.

\section{Limitations of the Study:}

Considering the rate of femur surgery our sample size is less, for more precise result more patients should be included in study. Assessment of SVS is difficult as pain is subjective feeling so same intensity of pain can be graded differently among the patients. Assessment of SVS and total requirement other analgesic agent will get affected if patients having pain other than IT femur.

\section{References}

1. Griffiths R, Alper J, et al. Management of proximal femoral fractures 2011: Association of Anaesthetists of Great Britain and Ireland. Anaesthesia. 2012;67(1):85-98.

2. Farzad M, Layeghi F, Hosseini A, Dianat A. Investigate the Effect of Psychological Factors in Development of Complex Regional Pain Syndrome Type I in Patients with Fracture: A Prospective Study. J Hand Surg Asian Pac. 2018;23(4):554-61

3. Morgan \& Mikhalis, Clinical anesthesiology, Fifth edition. Chronic Pain management, Chapter 47:8:1023-1105.

4. Vincent J. Collins. Principal of anesthesia, vol.2, Third edition local anesthetic, 1231-81

5. National Institute for Health and Care Excellence. The management of hip fracture in adults. National Clinical Guideline Centre. 2011. https: //www.nice.org.uk/guidance/ $\operatorname{cg} 124$ (accessed 27 Jun 2017).

6. Miller R D, Eriksson L I, Fleisher L A. Miller's Anesthesia 8th Edition. Vol.1 Peripheral nerve block. Chapter 57:2014:4:1740-51.

7. Ensrud KE. Epidemiology of Fracture risk with advancing age. J Gerontol Series A. 2013;68(10):1236-42.

8. Bharti B. Mahajan's method in biostatistics for medical students and research workers. JP productions.2018;9(2):42-100.

9. Haddad FS, Williams RL. Femoral nerve block in extracapsular femoral neck fractures. J Bone Joint Surg Br.1995;77:6:922-3.

10. Fentahun TK, Endale G, and Hailu YT. Efficacy of fascia iliaca compartment nerve block as part of multimodal analgesia after surgery for femoral bone fracture. World J Emerg Med. 2015; 6:2: 142-46.

11. Hanna L, Gulati L, Graham A. Hindawi Publishing Corporation; 2014. The role of fascia iliaca blocks in hip fractures: A prospective casecontrol study and feasibility assessment of a junior-doctor-delivered service. ISRN Orthopedics;2014: Article ID 191306

12. Nie H, Yang Y, Wang Y, Liu Y, Zhao B and Luan B. Effects of continuous fascia iliaca compartment blocks for postoperative analgesia in patients with hip fracture. Pain Res Management. 2015;20:4:210-12.

13. Williams H, Paringe V, Shenoy S, Michaels P, Ramesh B. Standard preoperative analgesia with or without fascia iliaca compartment block for femoral neck fractures. J Orthop Surg (Hong Kong). 2016;24:1:315 .

14. Nicolai B, Billy B. K, Morten B, Mikkel B, Christian H. Fascia Iliaca Compartment Blockade for Acute Pain Control in Hip Fracture Patients: A Randomized, Placebo-controlled Trial. Anesthesiology. 2007; 106:773-78.

Copyright: (C) the author(s), 2020. It is an open-access article distributed under the terms of the Creative Commons Attribution License (CC BY 4.0), which permits authors to retain ownership of the copyright for their content, and allow anyone to download, reuse, reprint, modify, distribute and/or copy the content as long as the original authors and source are cited.

How to cite this article: Shekhrajka P, Patel DC. Comparison of Fascia Iliaca Block with Intravenous Paracetamol, as Postoperative Analgesic Method for Femur Surgery. Acad. Anesthesiol. Int. 2020;5(1):13-16.

DOI: dx.doi.org/10.21276/aan.2020.5.1.3

Source of Support: Nil, Conflict of Interest: None declared. 